\title{
Effective lattice Polyakov loop theory vs. full SU(3) Yang-Mills at finite temperature
}

\author{
G. Bergner, ${ }^{a}$ J. Langelage ${ }^{b}$ and O. Philipsen ${ }^{a}$ \\ ${ }^{a}$ Institut für Theoretische Physik, Goethe-Universität Frankfurt, \\ Max-von-Laue-Str. 1, 60438 Frankfurt am Main, Germany \\ ${ }^{b}$ Institute for Theoretical Physics, ETH Zürich, \\ CH-8093 Zürich, Switzerland \\ E-mail: g.bergner@physik.uni-frankfurt.de, ljens@phys.ethz.ch, \\ philipsen@th.physik.uni-frankfurt.de
}

Abstract: A three-dimensional effective theory of Polyakov loops has recently been derived from Wilson's Yang-Mills lattice action by means of a strong coupling expansion. It is valid in the confined phase up to the deconfinement phase transition, for which it predicts the correct order and gives quantitative estimates for the critical coupling. In this work we study its predictive power for further observables like correlation functions and the equation of state. We find that the effective theory correctly reproduces qualitative features and symmetries of the full theory as the continuum is approached. Regarding quantitative predictions, we identify two classes of observables by numerical comparison as well as analytic calculations: correlation functions and their associated mass scales cannot be described accurately from a truncated effective theory, due to its inherently non-local nature involving long-range couplings. On the other hand, phase transitions and bulk thermodynamic quantities are accurately reproduced by the leading local part of the effective theory. In particular, the effective theory description is numerically superior when computing the equation of state at low temperatures or the properties of the phase transition.

Keywords: Lattice Gauge Field Theories, Strong Coupling Expansion, Confinement, Wilson, 't Hooft and Polyakov loops

ARXIV EPRINT: 1312.7823 


\section{Contents}

$\begin{array}{llr}1 & \text { Introduction } & 1\end{array}$

2 The effective lattice Polyakov loop theory 2

3 Polyakov loop correlators and static quark free energy 3

3.1 Two-point correlators 3

3.2 Weak coupling expansion in the effective theory 5

3.3 Effective $T$-dependent string tension 6

4 Thermodynamic potentials and phase transitions $\quad 8$

4.1 The equation of state 8

4.2 Validity of the effective action for thermodynamics and phase transitions 11

5 Conclusions $\quad 12$

A The couplings of the effective action $\quad 13$

\section{Introduction}

The use of Polyakov loop models as a simplified effective description of the pure glue sector of QCD at finite temperature has a long history. This is based on the expectation that, around the deconfinement phase transition, the dynamics of Yang-Mills theory is governed by the degrees of freedom which also constitute the order parameter for the global symmetry breaking driving the transition. Once an appropriate model is at hand, it is easier to analyse than the original theory, both with analytic methods or with numerical simulations. The goal is to obtain an effective description of Yang-Mills theory and ultimately full QCD, which would allow to determine the phase diagram and physical properties of QCD at finite baryon density, where lattice QCD exhibits its sign problem. For a recent example and references see [1]. In more recent approaches the aim is to actually derive the effective Polyakov loop theory directly from Yang-Mills or QCD by perturbation theory [2], strong coupling expansions [3] (see also [4], where the same method has been applied to large $N_{c}$ ), Monte Carlo methods [5-8] or the functional renormalisation group [9].

The various techniques employed to construct the effective theory each have their advantages and disadvantages. Effective theories derived by weak or strong coupling methods are only valid in the deconfined or confined phase, respectively, and thus are complementary. Their advantage are analytic expressions between the effective and fundamental couplings, which make for economic and flexible use of the effective theory to arrive at predictions. The disadvantage is the systematic error introduced by truncating the expansions at finite 
order. Non-perturbative approaches, on the other hand, have the advantage to potentially work at all couplings and to give a valid description on both sides of the phase transition. However, in this case the couplings are only known numerically and have to be recomputed for every change in the parameters of the original theory. Moreover, any particular form of an effective action with a finite number of terms necessarily implies truncations in the space of effective couplings, and an estimate of the implied systematic error is often more difficult than in series expansions.

This paper is devoted to a study of the systematics of a three-dimensional effective lattice action for Yang-Mills theory derived from the four-dimensional Wilson action by the strong coupling expansion [3]. The one-coupling effective theory derived in that work gives the correct prediction for the order of the $\mathrm{SU}(2), \mathrm{SU}(3)$ deconfinement transitions as well as the corresponding critical temperature $T_{c}$ to about $10 \%$ accuracy in the continuum limit. Here we extend the comparison between the effective and full theory to correlation functions of Polyakov loops, i.e. the free energy of a static quark anti-quark pair, as well as thermodynamic functions.

This work is organized as follows. In the next section we summarise the derivation of the effective theory. It correctly reproduces all qualitative features and symmetries of the full theory as the continuum is approached. We consider two classes of observables: in section 3 correlation functions and the associated length or mass scales. We find that they cannot be predicted accurately from a truncated effective theory, due to the inherent sensitivity to long-range interactions. On the other hand, phase transitions and bulk thermodynamic quantities in section 4 are accurately reproduced by the leading local part of the effective theory.

\section{The effective lattice Polyakov loop theory}

The effective lattice Polyakov loop theory is defined starting from Wilson's lattice YangMills action on a $N_{s}^{3} \times N_{\tau}$ lattice by splitting the link integrations into a spatial and temporal part,

$$
\begin{aligned}
Z & =\int\left[\mathrm{d} U_{\mu}\right] e^{-S_{Y M}[U]} \equiv \int[\mathrm{d} W] e^{-S_{\mathrm{eff}}[W]}, \\
S_{\mathrm{eff}}[W] & =-\sum_{i=1}^{\infty} \bar{\lambda}_{i}\left(\beta, N_{\tau}\right) S_{i}[W] .
\end{aligned}
$$

The individual terms in the effective action, $S_{i}[W]$, depend on temporal Wilson lines, $W(\mathbf{x})=\prod_{\tau=1}^{N_{\tau}} U_{0}(\mathbf{x}, \tau)$, or their traces, the Polyakov loops $L_{i}=\operatorname{tr}\left[W\left(\mathbf{x}_{i}\right)\right]$, which are the remaining integration variables in the path integral. Note that, without truncations, the effective action is unique and exact. Since all spatial links, which are originally coupled by nearest neighbour interactions, were integrated over, the effective action is of long-range type, irrespective of the way it is determined. It contains interactions of Polyakov lines at all distances, even a non-local form is allowed. Here we consider the case where the Boltzmann factor is expanded in a strong coupling expansion so that all link integrations 
can be performed analytically. The leading terms in the infinite volume limit read

$$
\begin{aligned}
S_{\text {eff }}[W]= & -\sum_{<i, j>} \ln \left(1+2 \lambda_{1}\left(\beta, N_{\tau}\right) \operatorname{Re} L_{i} L_{j}^{\dagger}\right)-\sum_{[k, l]} \ln \left(1+2 \lambda_{2}\left(\beta, N_{\tau}\right) \operatorname{Re} L_{k} L_{l}^{\dagger}\right) \\
& -\sum_{<<k, l>>} \ln \left(1+2 \lambda_{3}\left(\beta, N_{\tau}\right) \operatorname{Re} L_{k} L_{l}^{\dagger}\right)+\ldots,
\end{aligned}
$$

where $\langle i j\rangle$ denotes all pairs of nearest neighbours in the first term, $[k l]$ all pairs of next-to-nearest neighbours with distance $R / a=\sqrt{2}$, and $\langle<k l\rangle>$ all pairs with distance $R / a=2$. Without truncations, the action consists of infinitely many, generically not bilinear, terms with Wilson lines to all powers, all distances and in all representations, where the latter are a remnant of our preferred computational method, the character expansion. The higher representations can be converted back into products of the fundamental one, i.e. we may choose to work solely with fundamental loops to arbitrary powers. Note that in eq. (2.2) we have resummed higher powers of nearest neighbour interactions and nextto-nearest neighbours into a logarithm. This summation of an infinite number of terms redefines the couplings and improves the convergence behaviour, as discussed in detail in section 4 .

Using the strong coupling expansion, the terms in the effective action are naturally ordered by the lowest power of $\beta$ at which the corresponding effective coupling enters. Usually we express the effective couplings in terms of the fundamental character expansion coefficient $u=u(\beta)=\beta / 18+O\left(\beta^{2}\right)$, which shows better convergence. The relation between $u$ and $\beta$ can be computed to arbitrary precision, hence we can use them synonymously. A complete list of the couplings used in this work is summarized in the appendix, eq. (A.1) and eq. (A.2). As these expressions show, higher order couplings are parametrically suppressed with growing $N_{\tau}$, which corresponds to finer lattices at fixed temperature $T=1 /\left(a N_{\tau}\right)$.

Regardless of the truncation, the effective theory exhibits centre symmetry by construction and its spontaneous breaking at finite temperature. In [3] it was found that the theory truncated to the leading nearest neighbour interaction correctly predicts the different orders of the deconfinement phase transition for $\mathrm{SU}(2)$ and $\mathrm{SU}(3)$. Moreover, its predicted critical couplings for the phase transition agree with those from Monte Carlo simulations of the full theory to better than $10 \%$ accuracy for a wide range of temporal lattice sizes, $N_{\tau} \leq 16$, cf. table 1 . With an appropriate scale setting by means of a known $4 \mathrm{~d}$ beta-function [13], this permits the calculation of the deconfinement temperature $T_{c}$ in the continuum limit with similar accuracy [14]. In the following sections we investigate the predictive power of the effective theory for correlation functions and bulk thermodynamic quantities.

\section{Polyakov loop correlators and static quark free energy}

\subsection{Two-point correlators}

The spectrum of a theory is encoded in its correlation functions. A natural testing ground for the effective action are thus Polyakov loop correlators. Their exponential decay repre- 


\begin{tabular}{|c|c|c|c|}
\hline$N_{\tau}$ & $\lambda_{1}$ & $\left(\lambda_{1}, \lambda_{2}\right)$ & $4 \mathrm{~d} \mathrm{YM}$ \\
\hline 2 & $5.1839(2)$ & $5.0174(4)$ & $5.10(5)$ \\
3 & $5.8488(1)$ & $5.7333(3)$ & $5.55(1)$ \\
4 & $6.09871(7)$ & $6.0523(1)$ & $5.6925(2)$ \\
6 & $6.32625(4)$ & $6.32399(3)$ & $5.8941(5)$ \\
8 & $6.43045(3)$ & $6.43033(2)$ & $6.001(25)$ \\
10 & $6.49010(2)$ & $6.49008(2)$ & $6.160(7)$ \\
12 & $6.52875(2)$ & $6.52874(1)$ & $6.268(12)$ \\
14 & $6.55584(2)$ & $6.55583(1)$ & $6.4488(59)$ \\
16 & $6.57588(1)$ & $6.57587(1)$ & $6.5509(39)$ \\
\hline
\end{tabular}

Table 1. Critical couplings $\beta_{c}$ for $\mathrm{SU}(3)$ for the one- and two-coupling effective theories compared to simulations of the $4 \mathrm{~d}$ theory [10-12].
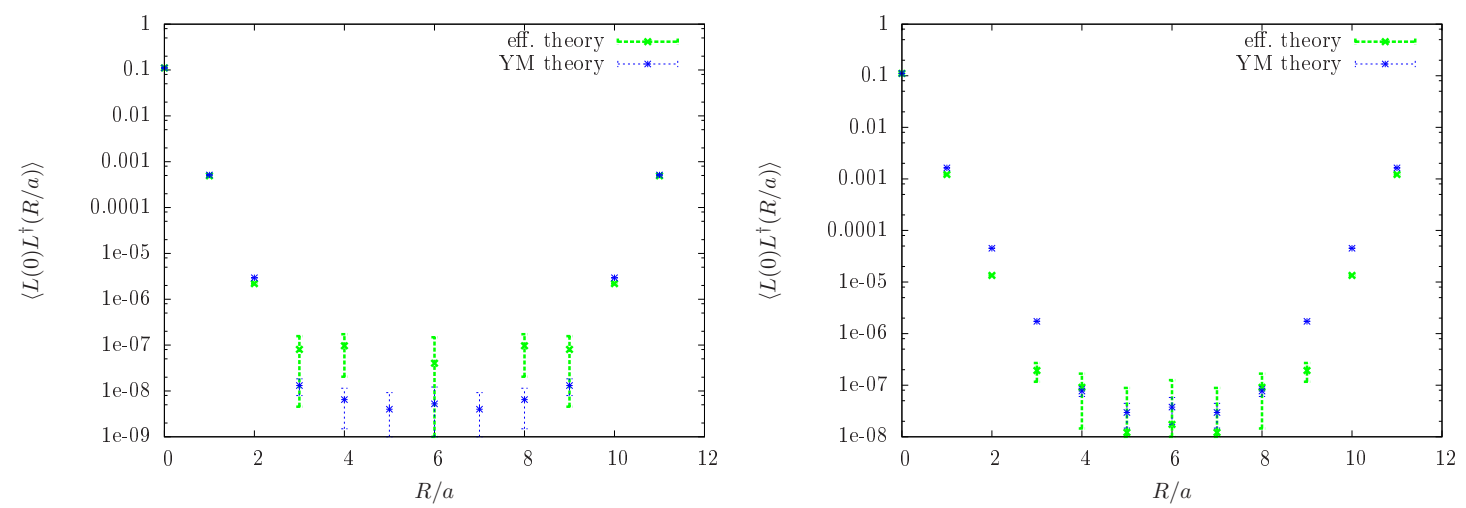

Figure 1. Comparison of the Polyakov loop correlator between the one-coupling effective theory and full Yang-Mills theory at $\beta=5.0$ (left) and $\beta=5.4$ (right). Both simulations were done on a $6 \times 12^{3}$ lattice.

sents the (unrenormalised) free energy of a static quark anti-quark pair [15],

$$
\left\langle L(R) L^{\dagger}(0)\right\rangle=e^{-F(R, T) / T} .
$$

A direct comparison of on-axis correlators between the one-coupling effective theory and full Yang-Mills is shown in figure 1 for two different values of the lattice coupling $\beta$. Note that in the full Yang-Mills simulation an algorithm for exponential error reduction [16] was employed, whereas the data for the effective theory have been obtained only with the comparably small improvement of a multi-hit algorithm. Quantitative agreement is observed for short lattice distances $R / a=0-2$ for $\beta=5.0$, while the effective theory data still follow the general shape of the full correlator, but start to quantitatively deviate for $R / a \geq 2$ at the larger coupling $\beta=5.4$.

Note that the correlators are systematically smaller, i.e. the corresponding free energies are larger in the effective theory. This is shown in continuum units in figure 2, with deviations increasing with distance. The off-axis correlators are also included in this figure. These are a measure for the breaking of rotational invariance by the lattice discretisation, which appears to be amplified in the effective theory compared to the full Yang-Mills theory. 

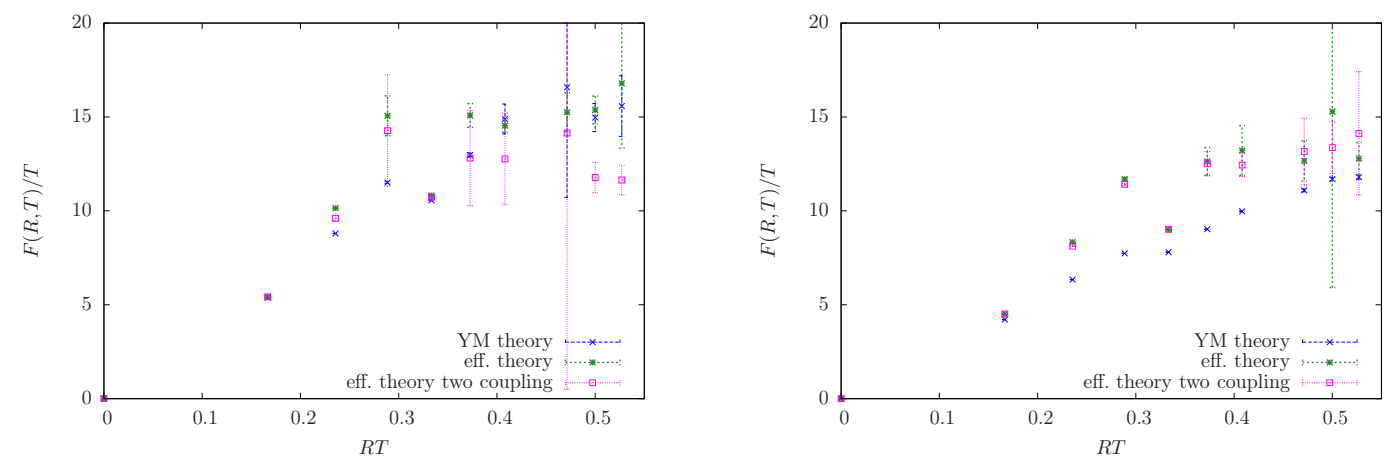

Figure 2. Free energy of a static quark-antiquark pair for full Yang-Mills and the effective theory with one and two coupling constants at $\beta=5.0$ (left) and $\beta=5.4$ (right) on $12^{3} \times 6$. This comparison includes also off-axis correlators.
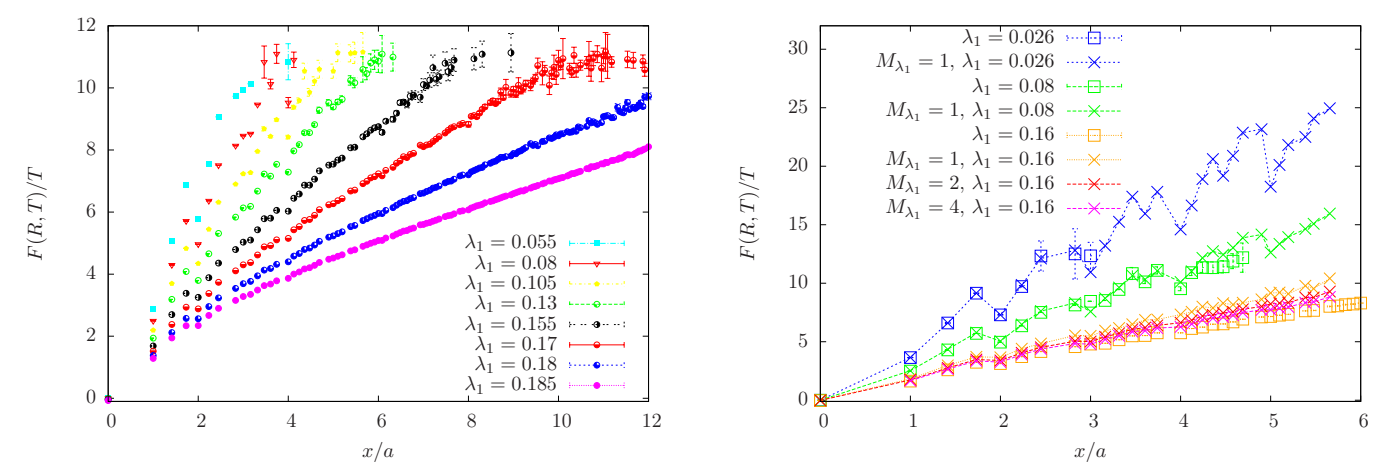

Figure 3. Left: free energy for different values of $\lambda_{1}$ in the effective theory on a $32^{3}$ lattice. The rotational invariance is restored at larger values of the coupling. Right: numerical simulations are compared to analytic results of the small $\lambda_{1}$ expansion, eq. (3.2).

This effect gets alleviated when also the next-to-nearest neighbour coupling is included in the effective theory. However, the improvement is small because of the smallness of the effective coupling, with values of $\lambda_{2}(\beta=5.0)=1.9 \times 10^{-5}$ and $\lambda_{2}(\beta=5.4)=5.6 \times 10^{-5}$, when using its strong coupling expansion eq. (A.2). On the other hand, when the effective coupling $\lambda_{1}$ (or equivalently $\beta$ ) is raised, rotational invariance gets restored also in the onecoupling theory, as figure 3 (left) illustrates, i.e. the effective action eventually reproduces the continuum symmetries. In this regime of larger $\lambda_{1}$ just below its critical value, which corresponds to larger $\beta$ and hence finer lattices, it is also possible to distinguish between a linear part at large distances and a Coulomb part at short distances.

\subsection{Weak coupling expansion in the effective theory}

Because of the smallness of the effective couplings, it is natural to consider perturbation theory for the effective action. Indeed, the correlator of Polyakov loops can be expressed as a power series in the coupling truncated at $M_{\lambda_{1}}$,

$$
\left\langle L(R) L^{\dagger}(0)\right\rangle \approx \sum_{n=1}^{M_{\lambda_{1}}} N_{n}(R / a) \lambda_{1}^{l_{n}(R / a)} .
$$




\begin{tabular}{|c|c|c|c|c|c|c|c|c|}
\hline$R / a$ & $N_{1}$ & $l_{1}$ & $N_{2}$ & $l_{2}$ & $N_{3}$ & $l_{3}$ & $N_{4}$ & $l_{4}$ \\
\hline 0 & 1 & 0 & 0 & 0 & 0 & 0 & 24 & 4 \\
1 & 1 & 1 & 4 & 3 & 8 & 4 & 76 & 5 \\
1.41421 & 2 & 2 & 18 & 4 & 12 & 5 & 316 & 6 \\
1.73205 & 6 & 3 & 60 & 5 & 54 & 6 & 1128 & 7 \\
2 & 1 & 2 & 12 & 4 & 8 & 5 & 240 & 6 \\
2.23607 & 3 & 3 & 49 & 5 & 22 & 6 & 909 & 7 \\
2.44949 & 12 & 4 & 178 & 6 & 98 & 7 & 3648 & 8 \\
2.82843 & 6 & 4 & 148 & 6 & 44 & 7 & 2918 & 8 \\
3 & 1 & 3 & 24 & 5 & 8 & 6 & 588 & 7 \\
3.16228 & 4 & 4 & 108 & 6 & 30 & 7 & 2398 & 8 \\
3.31662 & 20 & 5 & 444 & 7 & 158 & 8 & 10160 & 9 \\
3.4641 & 90 & 6 & 1872 & 8 & 720 & 9 & 43236 & 10 \\
3.60555 & 10 & 5 & 361 & 7 & 74 & 8 & 8253 & 9 \\
3.74166 & 60 & 6 & 1524 & 8 & 472 & 9 & 36242 & 10 \\
4 & 1 & 4 & 40 & 6 & 8 & 7 & 1260 & 8 \\
\hline
\end{tabular}

Table 2. A table of the expansion coefficients of the correlation function eq. (3.2). Only the shortest distances are listed in this table.

If the distance $R / a$ is greater than zero, $l_{1}$ is the "taxi-driver" distance on the lattice, i.e. the minimal number of links connecting the two correlated points. The coefficient functions $N_{n}$ count the number of possible paths of length $l_{n}$ that connect the correlated points. They have been estimated with a numerical algorithm and are summarized in table 2. Figure 3 (left) illustrates how for small values of $\lambda_{1}$, i. e. at strong coupling, this analytic result fully reproduces the numerical simulations of the effective model. With growing values of $\lambda_{1}$ and larger distances, higher orders in the expansion become important. At the largest $\lambda_{1}$ value shown in figure 3 , which corresponds at $N_{\tau}=4$ to $\beta \approx 6$, the fourth order expansion is still a good approximation for the short distance correlation.

\subsection{Effective $\boldsymbol{T}$-dependent string tension}

The linear part of the free energy corresponds to a temperature dependent effective string tension, which arises from the Boltzmann average over the linear pieces of the excitations of the static potential. This string tension decreases with $\lambda_{1}$ (or $\beta$ ), which is tantamount to increasing temperature at fixed $N_{\tau}$, in accord with full Yang-Mills theory $[17,18]$. To make the comparison quantitative, we fit our correlator in continuum units with the same ansatz used in [17] (details of the functional form are inspired by string models valid at large distances),

$$
\begin{aligned}
\frac{F(R, T)}{T}= & v_{0}+\frac{1}{2} \ln \left(1+(2 R T)^{2}\right)+\left[\frac{\pi}{12}-\frac{1}{6} \arctan (2 R T)\right] \frac{1}{R T} \\
& +\left[\frac{\sigma(T)}{T^{2}}-\frac{\pi}{3}+\frac{2}{3} \arctan \left(\frac{1}{2 R T}\right)\right] R T .
\end{aligned}
$$



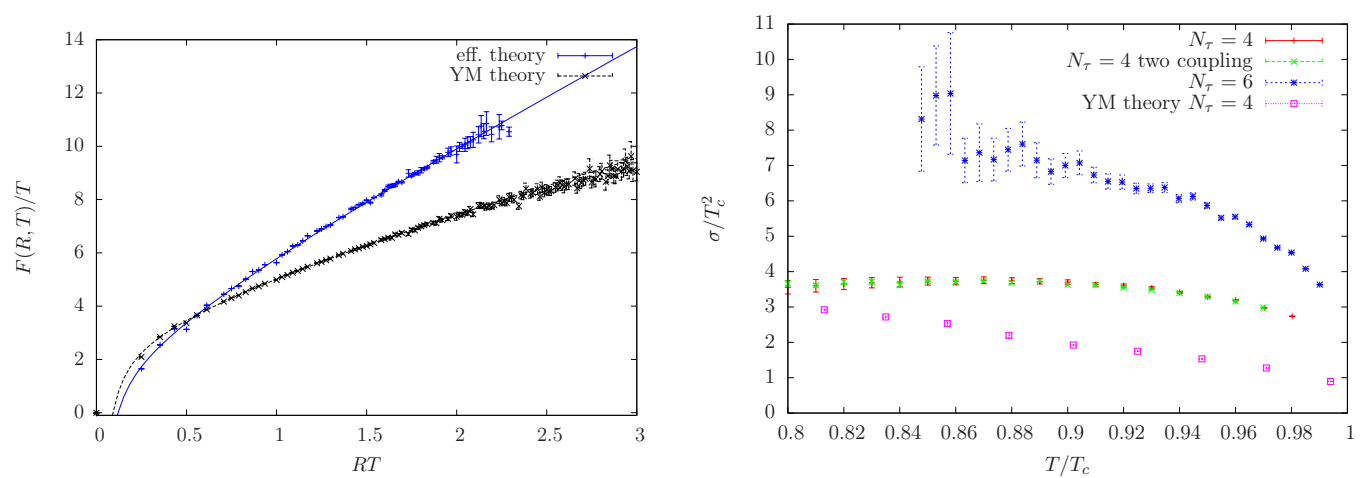

Figure 4. Left: free energy from the effective one-coupling theory at $T=90 \%\left(T_{c}\right)_{\text {eff }}$ on $32^{3} \times 4$ compared to Yang-Mills theory. The error bars include a systematic error as the difference between the $O\left(u^{10}\right)$ and $O\left(u^{9}\right)$ truncation of eq. (A.1). Right: effective temperature dependent string tension. The Yang-Mills data is taken from [18].

At short distances $R<T^{-1}$ the temperature effects disappear and the free energy is dominated by its ground state, the static potential of the vacuum,

$$
F(R, T) \stackrel{R \rightarrow 0}{\longrightarrow} V(R)=c_{1}+\frac{c_{2}}{R}+\sigma R
$$

Data from the effective theory close to the phase transition, $T=0.90 T_{c}$ are shown in figure 4 (left). They are well described by the finite temperature fit in the long distance region while being also compatible with the corresponding vacuum fit function appropriate for short distances. Thus, the qualitative features of the free energy of Yang-Mills theory are reproduced. However, the numerical values for the temperature dependent string tension for $N_{\tau}=6$ are significantly overestimated. A collection of fit results in comparison with the full answer is shown in figure 4 (right). We see that on $N_{\tau}=4$ the results are closer to the true answer and that the effective theory prediction seems to grow with $N_{\tau}$.

This incorrect scaling behaviour of the effective string tension is an artefact of the onecoupling theory. For all parirings $\left(\beta, N_{\tau}\right)$ with constant effective coupling $\lambda_{1}\left(\beta, N_{\tau}\right)$ the correlators as a function of distance in lattice units are the same. At large $R$ this implies that for two different $N_{\tau}$ with the same value of $\lambda_{1}$ the string tensions are related by

$$
\left(\frac{\sigma\left(T_{1}\right)}{T_{1}^{2}}+\frac{\pi}{3}\right) \frac{1}{\left(N_{\tau}\right)_{1}}=\left(\frac{\sigma\left(T_{2}\right)}{T_{2}^{2}}+\frac{\pi}{3}\right) \frac{1}{\left(N_{\tau}\right)_{2}},
$$

where the temperatures are determined by the corresponding $N_{\tau}$ and $\beta$. (The scale is set such that $T\left(\left(\lambda_{1}\right)_{c}\right)=T_{c}$ for all $\left.N_{\tau}\right)$. This forces the string tension to scale approximately with $N_{\tau}$ in the region close to $T_{c}$. The solution is an effective theory with more than one coupling constant. Then there is a critical (hyper-)surface and at each $N_{\tau}$ the phase transition can occur at a different values of the coupling constants. Our strong coupling result for the next-to-nearest neighbour interaction alone is however too small for a significant change of the string tension close to the phase transition.

One can understand qualitatively, why the value of the string tension cannot be correctly predicted by an effective theory with only a few couplings. We have already seen that 
the correlators are increasingly underestimated as the correlation distance in lattice units grows. A fixed distance in physical units contains more and more lattice spacings as the lattice gets finer. Correspondingly, the effective theory with a fixed number of couplings covers an ever smaller contribution to the correlator at fixed distance correctly. While the higher order couplings do become parametrically smaller $\sim u^{n N_{\tau}+m}$, eq. (A.1), a rapidly increasing number of them contributes to a correlator at distance $R / a$. Moreover, their apparent suppression with $N_{\tau}$ cancels in the contribution to the free energy. This can be demonstrated rather precisely by considering the strong coupling expansion of the vacuum string tension, i.e. the limit $N_{\tau} \rightarrow \infty$ at fixed $\beta$. The expansion starting from the $4 \mathrm{~d}$ Yang-Mills theory is well known [19] (for a finite temperature version, see [20]),

$$
\left.a^{2} \sigma\right|_{\mathrm{YM}}=-\ln u-4 u^{4}-12 u^{5}+10 u^{6}+O\left(u^{7}\right)
$$

On the other hand, expanding the $3 \mathrm{~d}$ effective theory in powers of the effective coupling constant $\lambda_{1}$ we obtain from the on-axis correlator

$$
\left.a^{2} \sigma\right|_{\text {eff.th. }}=-\frac{1}{N_{\tau}} \ln \left(\lambda_{1}\right)-\frac{2 \lambda_{1}}{N_{\tau}}+\ldots=-\ln u-4 u^{4}-12 u^{5}+14 u^{6}+O\left(u^{8}\right) .
$$

Only the first two terms agree with the expansion of the full theory. Note how the $N_{\tau^{-}}$ dependence of $\lambda_{1}$ is cancelled by an explicit $N_{\tau}$-dependence, while the second term is, as $\lambda_{1}$ itself, exponentially suppressed for increasing $N_{\tau}$. If we also include $\lambda_{3}$, the coupling for on-axis neighbours at distance $R / a=2$, we obtain instead

$$
\begin{aligned}
\left.a^{2} \sigma\right|_{\text {eff.th. }} & =-\frac{1}{N_{\tau}} \ln \left(\lambda_{1}\right)-\frac{2 \lambda_{1}^{2}}{N_{\tau}}-\frac{\lambda_{3} \lambda_{1}^{-2}}{N_{\tau}}+\sum_{n=2}^{\frac{1}{2}(R / a-1)} c_{n} \lambda_{3}^{n} \lambda_{1}^{-2 n} \ldots \\
& =-\ln u-4 u^{4}-12 u^{5}+10 u^{6}+O\left(u^{8}\right) .
\end{aligned}
$$

The detailed form of the last term in the first line depends on whether $R / a$ is even or odd, but the number of terms in the sum scales with $R / a$. Because of the $N_{\tau}$-dependence of the couplings, the second term is less significant than the third one for larger $N_{\tau}$. The leading contribution of the third term is $4 u^{6}$, such that the string tension is now correctly reproduced through order $u^{6}$. Correspondingly, the coefficients of higher orders receive more and more contributions from long-range couplings.

We conclude that the long-range interactions may not be neglected in the computation of correlation functions within the effective theory. Contrary to the effective couplings themselves, their contribution to correlators are not suppressed by $N_{\tau}$ and without them the coefficients of the strong coupling expansion of correlators are incomplete. While we have used the strong coupling expansion to show this, we stress that the conclusion is independent of the way the effective theory is determined or used, and in complete agreement with the non-perturbative observations made in [7].

\section{Thermodynamic potentials and phase transitions}

\subsection{The equation of state}

In this section we test the description of bulk thermodynamic quantities by the effective theory, which are all derived from the partition function directly. The fundamental ingre- 
dient to the equation of state is the free energy density in units of the temperature. In homogeneous systems it is related to the pressure $p$ as

$$
\frac{f}{T^{4}}=\frac{-p}{T^{4}}=-\frac{\ln Z}{V T^{3}} .
$$

For the correct renormalisation the divergent zero temperature part has to be subtracted.

In order to judge the quality of the effective theory, it is again instructive to consider the strong coupling expansion for different versions of the action,

$$
\text { 4d YM: } \quad f\left(u, N_{\tau}\right)=-\frac{6}{N_{\tau}} u^{4 N_{\tau}}+\ldots,
$$

$$
\begin{aligned}
\text { eff. theory, linear action: } & f\left(\lambda_{1}\left(u, N_{\tau}\right)\right)=-\frac{3}{N_{\tau}} \lambda_{1}^{2}+\ldots=-\frac{3}{N_{\tau}} u^{2 N_{\tau}}+\ldots, \\
\text { eff.theory, log. action: } & f\left(\lambda_{1}\left(u, N_{\tau}\right)\right)=-\frac{6}{N_{\tau}} \lambda_{1}^{4}+\ldots=-\frac{6}{N_{\tau}} u^{4 N_{\tau}}+\ldots .
\end{aligned}
$$

Here the full action is the ordinary strong coupling expansion without the detour of the effective theory $[21,22]$, the log. action corresponds to the first term of eq. (2.2) and the linear action to its leading term in $\lambda_{1}$ only. The explicit comparison reveals that the resummation of higher power terms into the logarithm is necessary to correctly reproduce the leading term of the full theory.

In a lattice simulation all expectation values are normalised on the partition function, which thus cannot be calculated directly. The free energy density is computed indirectly through its derivative with respect to the coupling constant $\beta$, which then has to be integrated over [23-25],

$$
\left.\frac{f}{T^{4}}\right|_{\beta_{0}} ^{\beta}=-\int_{\beta_{0}}^{\beta} d \beta^{\prime} \Delta S\left(\beta^{\prime}\right),
$$

with the interaction measure

$$
\Delta S(\beta)=\frac{1}{T^{4}}\left(\left.\frac{T}{V} \frac{d \ln Z}{d \beta}\right|_{T}-\left.\frac{T}{V} \frac{d \ln Z}{d \beta}\right|_{T=0}\right)=\frac{6 N_{\tau}^{4}}{N_{c}}\left(\left.\langle\operatorname{Re} P\rangle\right|_{T}-\left.\langle\operatorname{Re} P\rangle\right|_{T=0}\right) .
$$

Thus, all information of the equation of state is encoded in $\Delta S$. Computationally, this is a simple subtraction of two plaquette $(P)$ expectation values averaged over all orientations and volume. Finite temperature $T$ and $T=0$ are represented in terms of a $N_{\tau} \times N_{s}^{3}$ and a $N_{s}^{4}$ lattice with $N_{s}=4 N_{\tau}$.

The strong coupling expansion for $\Delta S$ can be obtained from the series for the pressure [3],

$$
\Delta S=N_{\tau}^{4} \frac{d\left(a^{4} p\right)}{d \beta}=N_{\tau}^{4} K\left(u, N_{\tau}\right) \frac{d u}{d \beta},
$$

where for $N_{\tau}=2,4$ we have

$$
\begin{aligned}
K\left(u, N_{\tau}=4\right)= & 24 u^{15}+1458 u^{17}-5643 u^{18}+9945 u^{19}-\frac{201285}{4} u^{20} \\
& +\frac{360638553}{1024} u^{21}-\frac{8627830587}{10240} u^{22}+\frac{6648458901}{5120} u^{23} \\
K\left(u, N_{\tau}=2\right)= & 24 u^{7}+270 u^{9}-1485 u^{10}+3315 u^{11}-\frac{4563}{4} u^{12}+\frac{126411873}{5120} u^{13} \\
& -\frac{221629365}{2048} u^{14}+\frac{648558969807}{5242880} u^{15}
\end{aligned}
$$


This can be compared with the data of the effective Polyakov loop action and the full theory. In the effective theory we compute

$$
\begin{aligned}
\Delta S(\beta)= & \frac{1}{T^{4}} \sum_{n}\left(\left.\frac{T}{V} \frac{d \ln Z}{d \lambda_{n}} \frac{d \lambda_{n}}{d u}\right|_{\left\{\lambda_{n}=\lambda_{n}\left[N_{\tau}, u\right]\right\}}-\left.\frac{T}{V} \frac{d \ln Z}{d \lambda_{n}} \frac{d \lambda_{n}}{d u}\right|_{\left\{\lambda_{n}=\lambda_{n}\left[N_{s}, u\right]\right\}}\right) \frac{d u}{d \beta} \\
= & 3 N_{\tau}^{4}\left(\left.\frac{1}{N_{\tau}}\left\langle R_{1}\left(\left\{\lambda_{i}\right\}\right)\right\rangle \frac{d \lambda_{1}}{d u}\right|_{\lambda_{1}=\lambda_{1}\left[N_{\tau}, u\right]}-\left.\frac{1}{N_{s}}\left\langle R_{1}\left(\left\{\lambda_{i}\right\}\right)\right\rangle \frac{d \lambda_{1}}{d u}\right|_{\lambda_{1}=\lambda_{1}\left[N_{s}, u\right]}\right) \frac{d u}{d \beta} \\
& +3 N_{\tau}^{4} \sum_{n=2}\left(\left.\frac{1}{N_{\tau}}\left\langle R_{n}\left(\left\{\lambda_{i}\right\}\right)\right\rangle \frac{d \lambda_{n}}{d u}\right|_{\lambda_{i}=\lambda_{i}\left[N_{\tau}, u\right]}-\left.\frac{1}{N_{s}}\left\langle R_{n}\left(\left\{\lambda_{i}\right\}\right)\right\rangle \frac{d \lambda_{n}}{d u}\right|_{\lambda_{i}=\lambda_{i}\left[N_{s}, u\right]}\right) \frac{d u}{d \beta} .
\end{aligned}
$$

where all expectation values are calculated on a $N_{s}^{3}$ lattice and $\left\langle R_{n}\right\rangle=1 /\left(3 N_{s}^{3}\right) d \ln Z / d \lambda_{n}$. In the one-coupling theory the last equation reduces to the first line with

$$
R\left(\lambda_{1}\right)=\frac{1}{3 N_{s}^{3}} \sum_{<i, j>} \frac{2 \operatorname{Re}\left(L_{i} L_{j}^{\dagger}\right)}{1+2 \lambda_{1} \operatorname{Re}\left(L_{i} L_{j}^{\dagger}\right)} .
$$

Again an expansion in the limit of small $\lambda_{1}$ provides a good check of the results. The expectation value of $R$ has a simple form in this limit, where it is dominated by the nearest-neighbour contribution between adjacent points $(i, j)$ on the lattice,

$$
\left\langle R_{1}\left(\lambda_{1}\right)\right\rangle \approx 2\left(4 \lambda_{1}^{3}+44 \lambda_{1}^{5}+O\left(\lambda_{1}^{6}\right)\right) .
$$

In this approximation finite volume corrections have been neglected.

We can now appreciate the difference to the situation for the string tension. Comparing eqs. $(4.7),(4.5)$, we see that they both have the same trivial $N_{\tau}^{4}$-dependence as a prefactor. Any other dependence on $N_{\tau}$ in eq. (4.7) is contained in the $\lambda_{n}$. Once again mixed polynomials in the $\lambda_{n}$ are needed to reproduce higher coefficients of the $4 \mathrm{~d}$ strong coupling expansion, but the power counting in $u$ can be based on that of the $\lambda_{n}$ directly, without cancellations of $N_{\tau}$-dependences as in the case of the string tension. The reason is that in the derivation of the effective theory the "observable" computed as a strong coupling series is the effective action itself, and thus the partition function. These analytic considerations are borne out by numerical simulations.

Unfortunately, the numerical determination of this quantity is quite difficult due to large cancellations in the subtraction, which implies a small signal-to-noise ratio. Particularly in the region of strong coupling (small $\beta$ ), $\Delta S$ drops exponentially causing a corresponding growth of the signal to noise ratio. For an overview and references, see [26]. It is then hard to bridge the gap between analytic strong coupling predictions [3] and simulations. In this regime the effective theory turns out to be very useful. Numerical results for $\Delta S$ as a function of the gauge coupling are shown in figure 5 for $8^{3} \times 2$ and $16^{3} \times 4$ lattices. The data demonstrate the advantage of the effective theory. Due to the larger statistics obtained in simple three dimensional simulations a much higher precision can be achieved than in the full theory. This allows to extend the considered $\beta$-range towards smaller values, in particular for higher $N_{\tau}$ where this is impossible in the full theory. This 

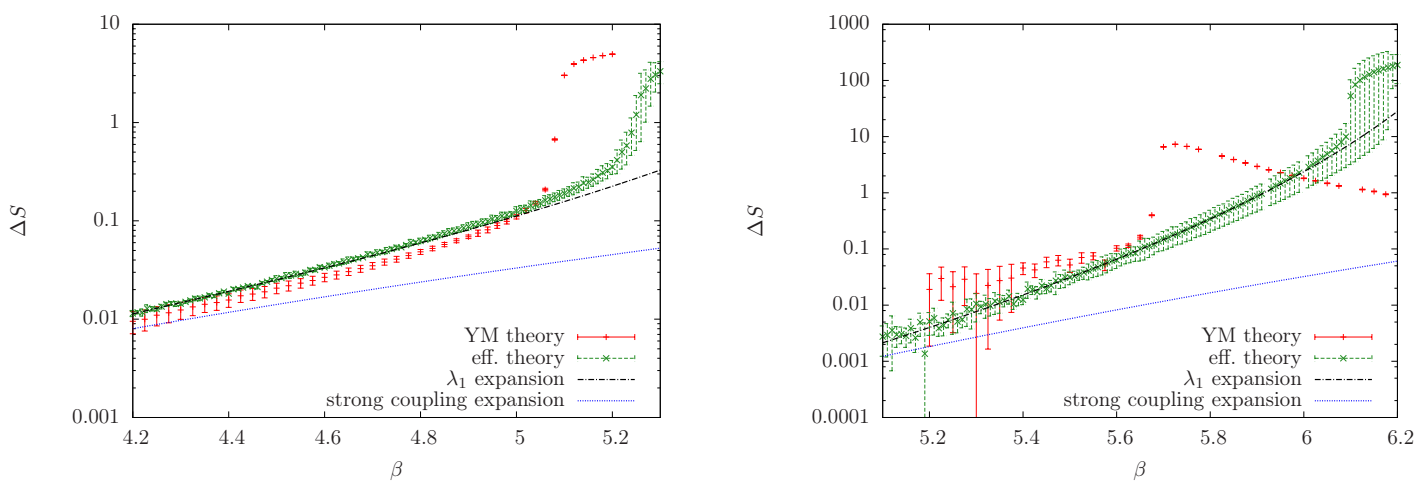

Figure 5. $\Delta S$ obtained in simulations of the full Yang-Mills theory and the effective theory. The left panel shows a lattice size of $8^{3} \times 2$ and $8^{3}$ respectively; the right panel a lattice size of $16^{3} \times 4$ and $16^{3}$. A systematic error is included as the difference of the $O\left(u^{9}\right)$ and $O\left(u^{10}\right)$ truncation in eq. (A.1). Also shown is the result of the small $\lambda_{1}$ expansion and the strong coupling expansion [22].

corresponds to the region of lower temperatures. We observe excellent quantitative agreement over a wide $\beta$-range. Deviations between full and effective theory predictions only set in at the deconfinement phase transition, with $\beta^{c}<\beta_{c}^{\text {eff }}$, cf. table 1.

In figure 5 we also compare with the fully analytic results of the strong coupling expansion and the expansion in small $\lambda_{1}$ within the effective theory. In a large $\beta$-range the small $\lambda_{1}$ expansion gives an excellent description. This indicates that for bulk quantities the short range interactions are dominant in the region well below the phase transition. Note that the $\lambda_{1}$-expansion is a much better approximation than the strong coupling series for the pressure in [22]. These two results converge, of course, in the strong coupling limit. At larger $\beta$ the use of the effective theory entails non-perturbative resummations compared to the straightforward strong coupling expansion, leading to an improved convergence towards the full theory.

\subsection{Validity of the effective action for thermodynamics and phase transitions}

While the critical couplings for the deconfinement transtion, table 1, have already been determined in [3], we would like to discuss here why the effective theory works so well for this observable. In statistical mechanics, a standard observable to locate a phase boundary is the generalised susceptibility of an observable $O(\mathbf{x})$,

$$
\chi_{O}=\int d^{3} x(\langle O(\mathbf{x}) O(0)\rangle-\langle O(\mathbf{x})\rangle\langle O(0)\rangle) .
$$

At a phase transition fluctuations are maximal, hence the peaks of susceptibilities define (pseudo-) critical couplings, whose finite size scaling moreover contains information about the order and universality class of the transition. The important observation is that, despite the integration over all distances, eq. (4.10) is a local observable for any theory with a mass gap. The correlators decay exponentially with distance,

$$
\langle O(\mathbf{x}) O(0)\rangle \sim \sum_{n} c_{n}^{2} e^{-E_{n}|\mathbf{x}|},
$$


with some energy eigenvalues $E_{n}$ and matrix elements $c_{n}$, such that the integral is dominated by the contact and short distance contributions. Moreover, at phase transitions the correlation length of a system either diverges (second order) or is maximal (first order and crossover), which implies that any scales smaller than the correlation length play either no or only a suppressed role. On the other hand, the behaviour of the correlation length is dictated by the symmetries and dimensionality of the theory. A similar reasoning applies for bulk thermodynamic quantities, which are derived from the partition function. The non-trivial quantity to compute in this case is the action, which again is local in the sense that couplings over larger distances are exponentially suppressed. Thus, a local effective action with the correct symmetries is capable to provide a good description of bulk thermodynamic quantities as well as phase transitions, even though it might be inaccurate for specific correlation functions or the spectrum of the theory.

\section{Conclusions}

We have systematically studied the predictive power of a three-dimensional effective Polyakov loop theory for Yang-Mills on the lattice, which has been derived previously by means of a strong coupling expansion. The effective theory has an infinite tower of interactions, with coupling between loops at all distances, of which only the first few are known analytically. Here we have tested the simplest version of the effective theory with just one (resummed) nearest neighbour coupling. Generally the accuracy of effective theory predictions depends on the observable where we distinguish to classes: observables characterised by explicit length scales, such as correlation functions, and bulk thermodynamic quantities based on the partition function or its local derivatives.

The description of correlation functions is found to be quantitatively accurate over short lattice distances only, $R / a \approx 0-2$. This is to be expected, since the number of neglected couplings increases rapidly with distance and the long-range interactions in the effective theory become increasingly important. The problem becomes more pronounced as the lattice spacing gets finer. Correlation functions at larger distances turn out to be systematically underestimated in this particular effective theory, resulting in an overestimate of the corresponding mass scales. In particular, the temperature dependent effective string tension extracted from the free energy of a static quark anti-quark pair is significantly too large close to the deconfinement transition.

On the other hand, bulk thermodynamic quantities like the equation of state and susceptibilities are quantitatively well described when approaching the deconfinement transition. This is because they are based on the partition function and thus the effective action itself, which becomes ultra-local in the continuum limit. Thus the effective theory is particularly useful for an economic determination of the phase structure of the underlying full theory. Because of the numerical ease with which accurate results can be obtained, the effective theory is superior for a description of the equation of state in the low temperature regime. Finally, these conclusions should carry over to the effective action describing dynamical QCD, derived by means of a hopping expansion [14], and its application to finite density phase transitions [27]. 


\section{Acknowledgments}

G.B. and O.P. are supported by the German BMBF, No. 06FY7100. J.L. is supported by SNF grant 200020-137920.

\section{A The couplings of the effective action}

The nearest neighbour interaction is parametrized by the coefficient $\lambda_{1}$. In this work we have employed the following series,

$$
\begin{aligned}
& \lambda_{1}\left(u, N_{\tau}=2\right)=u^{2} \exp \left[2 \left(4 u^{4}+12 u^{5}-18 u^{6}-36 u^{7}\right.\right. \\
& \left.\left.+\frac{219}{2} u^{8}+\frac{1791}{10} u^{9}+\frac{830517}{5120} u^{10}+\ldots\right)\right], \\
& \lambda_{1}\left(u, N_{\tau}=4\right)=u^{4} \exp \left[4 \left(4 u^{4}+12 u^{5}-14 u^{6}-36 u^{7}\right.\right. \\
& \left.\left.+\frac{295 u^{8}}{2}+\frac{1851 u^{9}}{10}+\frac{1035317 u^{10}}{5120}+\ldots\right)\right], \\
& \lambda_{1}\left(u, N_{\tau} \geq 6\right)=u^{N_{\tau}} \exp \left[N _ { \tau } \left(4 u^{4}+12 u^{5}-14 u^{6}-36 u^{7}\right.\right. \\
& \left.\left.+\frac{295}{2} u^{8}+\frac{1851}{10} u^{9}+\frac{1055797}{5120} u^{10}+\ldots\right)\right] .
\end{aligned}
$$

The coupling for next-to-nearest neighbours at distance $R / a=\sqrt{2}$ is

$$
\begin{aligned}
& \lambda_{2}\left(u, N_{\tau}=2\right)=u^{4}\left(2 u^{2}+6 u^{4}+31 u^{6}+\ldots\right), \\
& \lambda_{2}\left(u, N_{\tau}=4\right)=u^{8}\left(12 u^{2}+26 u^{4}+364 u^{6}+\ldots\right), \\
& \lambda_{2}\left(u, N_{\tau}=6\right)=u^{12}\left(30 u^{2}+66 u^{4}+\ldots\right),
\end{aligned}
$$

where the leading coefficient is given by $N_{\tau}\left(N_{\tau}-1\right)$ for all $N_{\tau}$.

The next-to-nearest neighbour interactions at distance $R / a=2$ is denoted by $\lambda_{3}$ and has the leading contribution

$$
\lambda_{3}\left(u, N_{\tau}\right)=4 N_{\tau} u^{2 N_{\tau}+6} .
$$

Open Access. This article is distributed under the terms of the Creative Commons Attribution License (CC-BY 4.0), which permits any use, distribution and reproduction in any medium, provided the original author(s) and source are credited.

\section{References}

[1] T.K. Herbst, M. Mitter, J.M. Pawlowski, B.-J. Schaefer and R. Stiele, Thermodynamics of QCD at vanishing density, arXiv:1308.3621 [INSPIRE].

[2] A. Vuorinen and L.G. Yaffe, Z(3)-symmetric effective theory for SU(3) Yang-Mills theory at high temperature, Phys. Rev. D 74 (2006) 025011 [hep-ph/0604100] [INSPIRE]. 
[3] J. Langelage, S. Lottini and O. Philipsen, Centre symmetric $3 d$ effective actions for thermal $\mathrm{SU}(N)$ Yang-Mills from strong coupling series, JHEP 02 (2011) 057 [Erratum ibid. 07 (2011) 014] [arXiv: 1010.0951] [INSPIRE].

[4] A.S. Christensen, J.C. Myers and P.D. Pedersen, Large-N lattice QCD and its extended strong-weak connection to the hypersphere, arXiv:1312.3519 [INSPIRE].

[5] C. Wozar, T. Kaestner, A. Wipf and T. Heinzl, Inverse Monte-Carlo determination of effective lattice models for $\mathrm{SU}(3)$ Yang-Mills theory at finite temperature, Phys. Rev. D 76 (2007) 085004 [arXiv:0704.2570] [INSPIRE].

[6] J. Greensite and K. Langfeld, Effective Polyakov line action from strong lattice couplings to the deconfinement transition, Phys. Rev. D 88 (2013) 074503 [arXiv:1305.0048] [InSPIRE].

[7] J. Greensite and K. Langfeld, Effective Polyakov line action from the relative weights method, Phys. Rev. D 87 (2013) 094501 [arXiv: 1301.4977] [INSPIRE].

[8] D. Smith, A. Dumitru, R. Pisarski and L. von Smekal, Effective potential for SU(2) Polyakov loops and Wilson loop eigenvalues, Phys. Rev. D 88 (2013) 054020 [arXiv:1307.6339] [INSPIRE].

[9] C.S. Fischer, L. Fister, J. Luecker and J.M. Pawlowski, Polyakov loop potential at finite density, arXiv:1306.6022 [INSPIRE].

[10] J. Fingberg, U.M. Heller and F. Karsch, Scaling and asymptotic scaling in the SU(2) gauge theory, Nucl. Phys. B 392 (1993) 493 [hep-lat/9208012] [INSPIRE].

[11] J.B. Kogut et al., Deconfinement and chiral symmetry restoration at finite temperatures in $\mathrm{SU}(2)$ and $\mathrm{SU}(3)$ gauge theories, Phys. Rev. Lett. 50 (1983) 393 [INSPIRE].

[12] A. Francis et al., Towards the continuum limit in transport coefficient computations, arXiv: 1311.3759 [INSPIRE].

[13] S. Necco and R. Sommer, The $N_{f}=0$ heavy quark potential from short to intermediate distances, Nucl. Phys. B 622 (2002) 328 [hep-lat/0108008] [INSPIRE].

[14] M. Fromm, J. Langelage, S. Lottini and O. Philipsen, The QCD deconfinement transition for heavy quarks and all baryon chemical potentials, JHEP 01 (2012) 042 [arXiv:1111.4953] [INSPIRE].

[15] L.D. McLerran and B. Svetitsky, Quark liberation at high temperature: a Monte Carlo study of SU(2) gauge theory, Phys. Rev. D 24 (1981) 450 [inSPIRE].

[16] M. Lüscher and P. Weisz, Locality and exponential error reduction in numerical lattice gauge theory, JHEP 09 (2001) 010 [hep-lat/0108014] [INSPIRE].

[17] O. Kaczmarek, F. Karsch, E. Laermann and M. Lutgemeier, Heavy quark potentials in quenched QCD at high temperature, Phys. Rev. D 62 (2000) 034021 [hep-lat/9908010] [INSPIRE].

[18] N. Cardoso and P. Bicudo, Lattice QCD computation of the $\mathrm{SU}(3)$ string tension critical curve, Phys. Rev. D 85 (2012) 077501 [arXiv:1111.1317] [InSPIRE].

[19] I. Montvay and G. Münster, Quantum fields on a lattice, Cambridge University Press, Cambridge U.K. (1994).

[20] F. Green, Strong coupling expansions for the string tension at finite temperature, Nucl. Phys. B 215 (1983) 83 [inSPIRE]. 
[21] J. Langelage, G. Munster and O. Philipsen, Strong coupling expansion for finite temperature Yang-Mills theory in the confined phase, JHEP 07 (2008) 036 [arXiv:0805.1163] [INSPIRE].

[22] J. Langelage and O. Philipsen, The pressure of strong coupling lattice QCD with heavy quarks, the hadron resonance gas model and the large-N limit, JHEP 04 (2010) 055 [arXiv: 1002.1507] [INSPIRE].

[23] J. Engels, F. Karsch, H. Satz and I. Montvay, Gauge field thermodynamics for the SU(2) Yang-Mills system, Nucl. Phys. B 205 (1982) 545 [inSPIRE].

[24] J. Engels, J. Fingberg, F. Karsch, D. Miller and M. Weber, Nonperturbative thermodynamics of SU(N) gauge theories, Phys. Lett. B 252 (1990) 625 [INSPIRE].

[25] G. Boyd et al., Thermodynamics of $\mathrm{SU}(3)$ lattice gauge theory, Nucl. Phys. B 469 (1996) 419 [hep-lat/9602007] [INSPIRE].

[26] O. Philipsen, The QCD equation of state from the lattice, Prog. Part. Nucl. Phys. 70 (2013) 55 [arXiv: 1207.5999] [InSPIRE].

[27] M. Fromm, J. Langelage, S. Lottini, M. Neuman and O. Philipsen, Onset transition to cold nuclear matter from lattice QCD with heavy quarks, Phys. Rev. Lett. 110 (2013) 122001 [arXiv: 1207.3005] [INSPIRE]. 